\title{
A conversation with P. Roy Vagelos
}

\section{7}

he biochemist P. Roy Vagelos is best known for leading Merck for 20 years, first as President of research and then later as $\mathrm{CEO}$ and Chairman of the Board. In his time at Merck, he revolutionized the way the company approached drug discovery, introduced the first statins to market, and organized the donation of ivermectin, the agent for control of river blindness, to any and all who need it for as long as it is needed. The full interview, including a story about almost being courtmartialed, can be seen on the JCI website, http://www.jci.org/kiosk/cgm.

JCI: What were you like as a kid?

Vagelos: I was born into a Greek immigrant family, and my first language was Greek. As I grew up, I was not at all interested in education, and only as I approached high school, did I start hearing the drum beat from my parents, who felt that higher education was important if I was not going to be in the family business. I went to high school in Rahway, New Jersey, and Rahway held the headquarters of Merck and, most importantly, the location of their major research laboratory. We had a small restaurant in Rahway, and our major customers were Merck people. So as a soda jerk and dishwasher in a small luncheonette, I heard about Merck, and specifically what was going on in research, from scientists and engineers. They had great ideas and loved what they were doing. They were passionate about their work, and that infected me. When it came time for college, I spoke with a number of them, and they suggested I go into chemistry. I was doing well at school by that time and went to the University of Pennsylvania, where I majored in chemistry.

JCI: From an interest in chemistry, what led you to medical school?

Vagelos: I was finishing my second year at Penn and met with an advisor who said that because I had taken so many courses in science, I could graduate in the next year if I took a few classes in summer school. As I was a scholarship student and rather concerned about expenditures, I took them up on it. I had to decide whether I was going to graduate and get a job doing chemistry, but at the last minute decided to go to medical school. I knew I had not taken any biology, so I spent my third year focusing on biology and just sneaked through. I went to medical school with the idea that chemistry would be useful in understanding what was happening in medicine.

JCI: But once you were there, patient care intrigued you.

Vagelos: I attended Columbia Medical School in New York City in 1950, mostly to change cities. I initially had difficulty with course work because I have a terrible memory; I function largely by feeling my way through things. The first course you're exposed to is human anatomy, and I almost

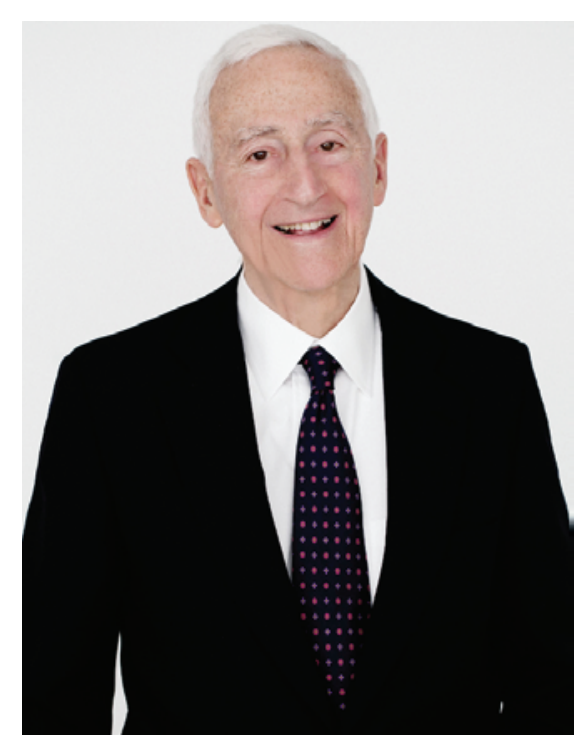

\section{Figure 1}

Roy Vagelos on March 28, 2014. Image credit: Alena Soboleva.

dropped out because I just could not learn the material; I went home at Christmas and considered not returning.

As we began biochemistry, I started to function better. Then I met with patients. I loved patient care and thought that this would definitely be my career; I became a complete convert when I went to Mass General Hospital as an intern in internal medicine. After two years at MGH, I had to pause because I had been deferred from the doctor draft, but by then I was absolutely sure that I wanted to do internal medicine and cardiology.

JCI: You moved to the NIH as part of the doctor draft?

Vagelos: I actually signed up for the army, but I was called by a friend who was at the $\mathrm{NIH}$ from MGH and invited me to visit.
I flew to Washington and visited the National Heart Institute. The deal was that I would spend half time taking care of heart patients and the other half I would be able to do research. I met Earl Stadtman, who was one of the two best-known biochemists at NIH. Earl was very soft-spoken, but as he spoke, he became very excited about his work. Even though I didn't really follow what he was saying, I ended up asking to work in his lab. He said, "You know, I've never worked with an MD." I replied, "Well, I have never worked with a PhD. So why don't we give it a try?"

Years later I had the opportunity to spend time in the lab of Jacques Monod, who was also an extraordinary person. Earl was a hard-nosed biochemist who believed only in facts and would not extrapolate in the broad sense. That's the atmosphere I was brought up in, but in the seventh year that I was at NIH, typically people take a sabbatical. I went to Paris to work with Jacques, who was so different in that he was a person who had dreams in science. He was very broad in knowledge and would take research observations and weave them into whole theories. I learned microbial genetics, almost as an apprentice to Jacques. However, I was very anxious to get back to what I was doing at NIH because we were on the verge of understanding fatty acid biosynthesis, and that work was actually more exciting to me.

JCI: After your time back at the NIH, you moved to Washington University as chair of Biochemistry.

Vagelos: I decided at NIH to become totally involved in biochemistry because I was pulled in by my own research. My patient care responsibilities had dropped enormously over the years, but I was concerned that I had given up my involvement in medicine. I thought going back to a medical school would be useful. Wash U was and is a great medical school, and one of my best postdocs [Phil Majerus] was from there. It was a wonderful move, as I had a blossoming of my research; much of the work that we did in fatty acids and lipids was carried out there. I restructured the graduate curriculum and started the MD$\mathrm{PhD}$ program, which turned out to be one of the strongest in the US.

JCI: If things were going so well, what motivated you to consider going to Merck - which you did in 1975? 
Vagelos: About that time, I was called by two major medical schools, Penn and University of Chicago, and asked to visit with search committees seeking a new dean. That was very depressing for me because the idea of leaving science at age 45 was abhorrent. I was then approached by Merck. I visited the research labs and found exciting people very interested in drug discovery, something I had never considered.

JCI: Among many triumphs in drug discovery while you were there, Merck brought the first statin to market. Can you tell me a little bit about your role in the upsets and then ultimately the triumph?

Vagelos: I suggested that one area that was ripe was the area of cholesterol biosynthesis. The total biosynthesis of cholesterol had just been worked out by Bloch and Lynen, and Brown and Goldstein were probing cholesterol regulation, so it was a project ready for a biochemical approach. Al Alberts, an old friend from NIH who accompanied me to Wash U and Merck, and I agreed that a cholesterol biosynthesis reductor would be one of our first projects. Our target (HMG-CoA reductase) had been shown to be the limiting reaction in cholesterol biosynthesis.

Just as we were starting, we heard of Akira Endo's work in Japan and the discovery of a naturally occurring statin (Compactin), so we were already behind in this competition. Merck had much experience in natural product studies, so Al looked for an inhibitor of HMG-CoA in fermentation broths as well as among chemicals from Merck chemists. Within a short time, Al came up with lovastatin. It was a reversible competitive inhibitor of HMG-CoA reductase. It was safe in animals, and we raced into the clinic. We were very excited to learn that lovastatin, like Compactin, was able to reduce cholesterol levels in humans.

JCI: Unfortunately, a rumor derailed you. Vagelos: A rumor that was devastating. We heard from Japan that the clinical studies of Compactin had stopped with no reason given, but there was a rumor that the compound caused tumors in animals. I got on the phone with the people at the company where Endo was working (Sankyo). They claimed, "It's an industry secret." We could get no information, but there was the possibility that our inhibitor might do a similar thing... we couldn't put humans at risk of potentially getting cancer. Clinical studies were immediately stopped.

JCI: It was the NIH who begged you to restart the clinical trials in high-risk patients.
Vagelos: The NIH and the FDA were believers in high cholesterol as a major risk factor. Here was a drug that could possibly help people dying because of their high blood cholesterol. They asked us to try again in high-risk patients: patients who had high blood cholesterol and coronary heart disease. Clinical studies showed that lovastatin reduced blood LDL cholesterol and was safe. In 1987, the first statin in the world was introduced by Merck. Some doctors believed in the cholesterol hypothesis and immediately started prescribing it; others were skeptical. So we carried out an outcome study with a second statin (simvastatin/Zocor) - the Scandinavian Simvastatin Survival Study. At the end of 5.5 years, they broke the blind and compared the 4,400 patients. They saw a reduction in death from any cause of $30 \%$, a reduction in death from heart attacks by $43 \%$, and a reduction in strokes by $30 \%$. The results of that one study changed hypothesis to fact and essentially revolutionized the treatment of heart disease. That was the beginning of the statin revolution.

JCI: You are also behind another revolution: a Merck scientist discovered that ivermectin, which was originally developed as an antiparasitic for livestock, could potentially prevent river blindness in humans. You later pioneered the donation of ivermectin to anyone who might need it for as long as it was needed, for free. This was the first true pharmaceutical philanthropy.

Vagelos: Bill Campbell, one of our scientists, demonstrated that ivermectin could kill many parasites with a potency 100 times greater than any known drug at the time. In 1981, Mohammed Aziz, who had been in the WHO in sub-Saharan Africa, told me about river blindness and noted there were 18 million people who were infected and going blind because of a parasite called Onchocerca volvulus, with no good drug to prevent it. He suggested we try ivermectin, which was put into a tablet form named Mectizan. Mohammed went off to Dakar, Senegal, where he tested the drug in some of these patients.

This parasite exists as an adult worm that lives in the skin. The males get to be about four to six inches, the females about sixteen to eighteen inches. They live together in lumps about the size of a small ping pong ball in the skin and produce microfilaria. The microfilaria also migrate into the eyes, where they cause inflammation and ultimately blindness. Mohammed gave one tablet of Mectizan to each patient; then he returned in a month, and the microfilaria were gone. He came back to Rahway very excited. We talked with the WHO specialists, who said the results were probably wrong because there's no way that a drug could eliminate all the parasites without causing serious side effects. We knew the results were correct, so we undertook a large development program, knowing that the people who might require Mectizan were among the poorest in the world.

JCI: Unfortunately, you couldn't get any governments to help you defray the cost.

Vagelos: Our marketing people thought we could find a price that was feasible, especially by selling it to governments. But the governments had different priorities, and neither African nor the US government had money in the budget, even though we were only talking about $\$ 2$ million at the start. By then, I was CEO, and I was meeting with the executive group trying to figure out how we were going to get this drug to the patients. The New York Times picked up the story. The magazine section had river blindness on the cover, and the story mentioned that Merck had a drug that was almost miraculous - and what was Merck going to do with it? So the pressure was on. Our executive group met and made a decision, then had a press conference in Washington to announce that the company would contribute Mectizan free to anyone in the world for as long as it was required.

I was invited back to Merck at the 25th anniversary of the program in 2012. I learned at that time that Merck treated about 200 million people that year for not only river blindness, but also elephantitis (another parasitic infection that could be controlled by Mectizan).

JCI: When some of the Merck workers were on strike in the 80 s, you rolled up your sleeves in the cafeteria and made sandwiches for those still working. So if you were not a scientist, an MD, or a pharmaceutical leader, what other vocation do you think you would have chosen? Would you be a soda jerk like in your youth?

Vagelos: That's really inconceivable. From early days, I saw how hard my parents worked in order that I receive a good education. My career could not have been anything other than biomedical science. One can make dramatic improvements in people's lives through science. I don't know what I would have done had it not been medical science. I can't understand why anybody would work in any other field.

\section{Ushma S. Neill}

